\title{
DETC99/DAC-8638
}

\section{SERIAL 5DOF MANIPULATORS: WORKSPACE, VOID, AND VOLUME DETERMINATION}

\author{
K Abdel-Malek \\ Department of Mechanical Engineering, \\ The University of lowa, lowa City, IA 52242 \\ Tel. (319) 335-5676 \\ amalek@engineering.uiowa.edu
}

\author{
Harn-Jou Yeh \\ Microtek, Inc. \\ Taiwan \\ hjyeh@microtek.com.tw
}

\author{
Nada Khairallah \\ Faculty of Engineering and Architecture \\ American University of Beirut \\ Beirut, Lebanon \\ malak@cyberia.net.lb
}

\begin{abstract}
Algorithms for identifying closed form surface patches on the boundary of 5DOF manipulator workspaces are developed and illustrated. Numerical algorithms for the determination of three- and four-DOF manipulator workspaces are available, but formulations for determining equations of surface patches bounding the workspace of five-DOF manipulators were never presented. In this work, constant singular sets in terms of the generalized variables are determined. When substituted into the vector function yield hyperentities that exist internal and external to the workspace envelope. The appearance of surfaces parametrized in three variables within the workspace requires further analysis pertaining to a coupled singular behavior and is also addressed. Previous results pertaining to bifurcation points that were unexplained are now addressed and clarified. Numerous examples are presented.
\end{abstract}

\section{INTRODUCTION}

Numerical methods for determining boundaries of workspaces of mechanisms and manipulators have been developed by a number of authors in recent years. These studies have also extended to the field of computer-aided design (Abdel-Malek and Yeh 1997a) where the computation of the swept volume is important to solid modeling. Similarly, in manufacturing, the $\mathrm{NC}$ verification of machining processes requires the computation of the workspace generated by the tool path on the workpiece. More recently, Cecarelli (1995) used an algebraic formulation of a workspace boundary to formulate design equations of three-revolute (3R) jointed manipulators and 4R manipulators (Cecarelli and Vinciquerra 1995). The benefit of this method is shown in the ability to determine holes and voids in the accessible output set. The same example treated by Cecarelli will be addressed here for validation purposes. Other works that have dealt with manipulator workspace are reported by Kumar (1985), and Emiris (1993) and Zhang et al. (1996).

Recently, Haug et al. (1996) formulated numerical criteria to find the workspace (called the accessible output set) of a general multi-degree-of-freedom system using a continuation method to trace boundary curves suitable for the study of both open- and closed-loop manipulators. The initial criteria for this computational method were presented by Haug et al. (1992) and Wang and Wu (1993). The algorithm computes tangent vectors at bifurcation points of continuation curves that define the boundary of manipulator workspaces. A crosssection of the workspace is obtained and boundary continuation curves are traced. The method was demonstrated for a closed-loop mechanism called the Stewart Platform (Luh et al. 1996). These curves are then assembled into a mesh that is enveloped by appropriate surface patches. This method has proved valid for determining the general shape of the accessible output set. The main difficulty is in determining the 
status of a singularity at points along continuation curves. Although singular behavior occurring at points along the curves is identified, this method is completely numerical and only traces boundary curves. It does not result in analytical surfaces bounding the accessible output set. A comparison between the numerical approach introduced by Haug and colleagues and the approach of the method presented here for lower DOF was discussed by Abdel-Malek et al. (1997).

The aim of this paper is to generalize the formulation for determining the workspace of the 3-DOF system presented elsewhere (Abdel-Malek and Yeh 1997b), to a general 5-DOF system and to explain previous results that remained unexplained. A formulation pertaining to a rank-deficiency criteria of the position Jacobian, including the effect of joint limits, is presented in section 2. First- and second-order criteria are introduced to delimit singular sets. These sets when substituted into the position vector of the end-effector yield parametric equations of surfaces. The appearance of surfaces parametrized in three generalized coordinates is addressed through second-order analysis in Section 3. The formulation is demonstrated through the analysis of spatial 5DOF examples in Section 4.

\section{FORMULATION}

Define $\mathbf{q}^{*} \in \mathfrak{R}^{n}$ as the vector of $n$-generalized coordinates characterizing a manipulator configuration. The vector function generated by a point on the end-effector of a serial arm written as a multiplication of rotation matrices and position vectors is expressed by

$\boldsymbol{\xi}\left(\mathbf{q}^{*}\right)=\left[\begin{array}{l}f\left(\mathbf{q}^{*}\right) \\ g\left(\mathbf{q}^{*}\right) \\ h\left(\mathbf{q}^{*}\right)\end{array}\right]=\sum_{i=1}^{i=n}\left[\prod_{j=1}^{j=i-1}{ }^{j-1} \mathbf{R}_{j}\right]^{i-1} \mathbf{p}_{i}$

where $\boldsymbol{\xi}\left(\mathbf{q}^{*}\right)=\left[\begin{array}{lll}x & y & z\end{array}\right]^{T}$ and $\mathbf{q}^{*}=\left[q_{1}, \ldots, q_{n}\right]^{T}$, and both ${ }^{i} \mathbf{p}_{j}$ and ${ }^{i} \mathbf{R}_{j}$ are defined using the Denavit-Hartenberg representation method (Denavit and Hartenberg 1955 and Fu et al. 1987) such that

$$
{ }^{i-1} \mathbf{R}_{i}=\left[\begin{array}{ccc}
\cos \theta_{i} & -\cos \alpha_{i} \sin \theta_{i} & \sin \alpha_{i} \sin \theta_{i} \\
\sin \theta_{i} & \cos \alpha_{i} \cos \theta_{i} & -\sin \alpha_{i} \cos \theta_{i} \\
0 & \sin \alpha_{i} & \cos \alpha_{i}
\end{array}\right]
$$

$$
\text { and }{ }^{(i-1)} \mathbf{p}_{i}=\left[\begin{array}{lll}
a_{i} \cos \theta_{i} & a_{i} \sin \theta_{i} & d_{i}
\end{array}\right]^{T}
$$

where $\theta_{i}$ is the joint angle from $\mathbf{x}_{i-1}$ axis to the $\mathbf{x}_{i}$ axis, $d_{i}$ is the shortest distance between $\mathbf{x}_{i-1}$ and $\mathbf{x}_{i}$ axes, $a_{i}$ is the offset distance between $\mathbf{z}_{i}$ and $\mathbf{z}_{i-1}$ axes, and $\alpha_{i}$ is the offset angle from $\mathbf{z}_{i-1}$ and $\mathbf{z}_{i}$ axes. The generalized variable is $q_{i}=d_{i}$ if the joint is prismatic and $q_{i}=\theta_{i}$ if the joint is revolute. The vector function $\boldsymbol{\xi}\left(\mathbf{q}^{*}\right)$ characterizes the set of all points inside and on the boundary of the workspace. The aim of this work is to determine the boundary to this set and to analytically represent it. At a specified position in space, Eq. 1 can be written as a constraint function

$$
\Omega\left(\mathbf{q}^{*}\right)=\left[\begin{array}{lll}
f\left(\mathbf{q}^{*}\right)-x & g\left(\mathbf{q}^{*}\right)-y & h\left(\mathbf{q}^{*}\right)-z
\end{array}\right]^{T}=\mathbf{0}
$$

Joint limits imposed in terms of inequality constraints in the form of $q_{i}^{L} \leq q_{i} \leq q_{i}^{U}$, where $i=1, \ldots n$ are transformed into equations (Haug et al. 1996) by introducing a new set of generalized coordinates $\lambda=\left[\lambda_{1}, \ldots, \lambda_{n}\right]^{T}$ such that

$$
\begin{gathered}
q_{i}=\left(\left(q_{i}^{L}+q_{i}^{U}\right) / 2\right)+\left(\left(q_{i}^{U}-q_{i}^{L}\right) / 2\right) \sin \lambda_{i} \\
i=1, \ldots, n
\end{gathered}
$$

These generalized coordinates $\lambda_{i}$ are called slack variables in the field of optimization. In order to include the effect of joint limits, it is proposed to augment the constraint equation $\boldsymbol{\Omega}\left(\mathbf{q}^{*}\right)$ with the parametrized inequality constraints such that

$$
\mathbf{H}(\mathbf{q})=\left[\begin{array}{c}
f\left(\mathbf{q}^{*}\right)-x \\
g\left(\mathbf{q}^{*}\right)-y \\
h\left(\mathbf{q}^{*}\right)-z \\
q_{i}-a_{i}-b_{i} \sin \lambda_{i}
\end{array}\right]=\mathbf{0} \quad i=1, \ldots n
$$

where $\quad \mathbf{q}=\left[\begin{array}{ll}\mathbf{q}^{* T} & \lambda^{T}\end{array}\right]^{T}$ is the vector of all generalized coordinates. Note that although $n-$ new variables $\left(\lambda_{i}\right)$ have been added, $n$-equations have also been added to the constraint vector function without loosing the dimensionality of the problem.

The Jacobian of the constraint function $\mathbf{H}(\mathbf{q})$ at a point $\mathbf{q}^{0}$ is the $(3+n) \times 2 n$ matrix

$$
\left[\mathbf{H}_{\mathbf{q}}\left(\mathbf{q}_{o}\right)\right]=\llbracket \partial \mathbf{H} / \partial \mathbf{q} \rrbracket
$$

where the subscript denotes a derivative. With the modified formulation including the parametrized inequality constraints, the Jacobian is expanded as

$$
\left[\mathbf{H}_{\mathbf{q}}\right]=\left[\begin{array}{c:c}
\boldsymbol{\xi}_{\mathbf{q}^{*}} & \mathbf{0} \\
\hdashline \mathbf{I} & \mathbf{q}_{\lambda}^{*}
\end{array}\right]
$$

where the notation $f_{q_{1}}$ denotes the partial derivative of $f$ with respect to $q_{1}$, and

$$
\left[\boldsymbol{\xi}_{\mathbf{q}^{*}}\right]=\left[\begin{array}{llll}
f_{q_{1}} & f_{q_{2}} & \ldots & f_{q_{n}} \\
g_{q_{1}} & g_{q_{2}} & \ldots & g_{q_{n}} \\
h_{q_{1}} & h_{q_{2}} & \ldots & h_{q_{n}}
\end{array}\right]
$$

and 


$$
\left[\mathbf{q}_{\lambda}^{*}\right]=\left[\begin{array}{ccc}
-\left(\left(q_{1}^{U}-q_{1}^{L}\right) / 2\right) \cos \lambda_{1} & 0 \\
0 & & -\left(\left(q_{2}^{U}-q_{2}^{L}\right) / 2\right) \cos \lambda_{2} \\
0 & & 0 \\
0 & & 0 \\
& \ldots & 0 \\
\ldots & 0 \\
\ldots & -\left(\left(q_{n}^{U}-q_{n}^{L}\right) / 2\right) \cos \lambda_{n}
\end{array}\right]
$$

is a diagonal block matrix, $\mathbf{0}$ is a $(3 \times n)$ zero matrix, and $\mathbf{I}$ is the identity matrix. The boundary to the workspace $\partial W$ (workspace envelope) is a subset of the workspace at which the Jacobian of the constraint function of Eq. 7 is row rank deficient (Kumar and Waldron 1981, Haug et al. 1996, AbdelMalek and Yeh 1997b); i.e.,

$$
\partial W \subset\left\{\operatorname{Rank} \mathbf{H}_{\mathbf{q}}(\mathbf{q})<k, \text { for some } \mathbf{q} \text { with } \mathbf{H}(\mathbf{q})=\mathbf{0}\right\}
$$

where $k$ is at least $(3+n-1)$. For an $n$-DOF system, the Jacobian $\mathbf{H}_{\mathbf{q}}\left(\mathbf{q}^{o}\right)$ is row-rank deficient if and only if one of the following conditions are satisfied.

\section{No Joints Reach their Limits}

If no joints have reached their limits, the diagonal sub-matrix $\left[\mathbf{q}_{\lambda}^{*}\right]$ is full row rank. Therefore, the only possibility for $\left[\mathbf{H}_{\mathbf{q}}\right]$ to be row-rank deficient is when the block matrix $\left[\xi_{q^{*}}\right]$ is row rank deficient. Define two independent subvectors of $\mathbf{q}^{*}$ as $\mathbf{p}$ and $\mathbf{u}$, as

$$
\mathbf{q}^{*}=\left[\begin{array}{ll}
\mathbf{p}^{T} & \mathbf{u}^{T}
\end{array}\right]^{T}, \text { where } \mathbf{p}, \mathbf{u} \in \mathbf{q}^{*} \text { and } \mathbf{p} \cap \mathbf{u}=\phi
$$

If $\mathbf{u} \in \mathbf{R}^{m}$ then $\mathbf{p} \in \mathbf{R}^{(n-m)}$.

Type I singularity set can be defined as $S^{(1)} \equiv\left\{\mathbf{p} \in \mathbf{q}^{*}: \operatorname{Rank}\left[\xi_{\mathbf{q}^{*}}\right]<3\right.$, for some constant subset of $\left.\mathbf{q}\right\}$

where $\mathbf{p}$ is within the specified joint limit constraints. The $(m \times n)$ block matrix $\left[\boldsymbol{\xi}_{\mathbf{q}^{*}}\right]$ is row rank deficient at least one, where $m=3$ for spatial and $m=2$ for planar manipulators. The rank of the $(m \times n)$ matrix is defined to be the order of the largest non-singular square sub-matrix which can be formed by selecting rows and columns of the upper corner matrix $\left[\xi_{\mathbf{q}^{*}}\right]$. In order to make the sub-matrix $\left[\xi_{\mathbf{q}^{*}}\right]$ rank deficient of order $(d)$ where $d=\operatorname{abs}(m-n)$, it is necessary to determine all sub-Jacobians. For a rank-deficiency $(d)$, the largest square sub matrix cannot have a larger dimension than $b=\max (n-d, m-d)$. Therefore, there must be $m ! /[(m-b) ! b !]$ possible independent rows that can be considered in a single square sub-matrix. Similarly, there are $n ! /[(n-b) ! b !]$ possible combinations of columns. Hence, there exists

$$
\eta=\frac{n !}{(n-b) ! b !} \cdot \frac{m !}{(m-b) ! b !}
$$

sub-Jacobians. Equating the determinants to zero yields $\eta$ number of equations to be simultaneously solved. Solutions to the $\eta$ equations are the singular sets of Type $\mathbf{I}$. This criterion is used to obtain square sub-Jacobians. Solutions of the resulting $\eta$ equations of all 3 conditions are sets of constant parameters denoted by $\mathbf{p}_{i}$ and characterized by

$\mathbf{p}_{i}=\left\{\left[\begin{array}{c}\operatorname{det}\left(\hbar_{i} \hbar_{j} \hbar_{k}\right)_{1} \\ \vdots \\ \operatorname{det}\left(\hbar_{i} \hbar_{j} \hbar_{k}\right)_{\eta}\end{array}\right]=\mathbf{0}\right.$, for $i, j, k=1, \ldots, n$ and $\left.i \neq j \neq k\right\}$
$i=1,2, \ldots, \beta$

where $\hbar_{i}$ denotes a column of $\left[\xi_{\mathbf{q}^{*}}\right]=\left[\hbar_{k}, \ldots, \hbar_{m}\right]$. For each $\mathbf{p}_{i}$, remaining variables are $\mathbf{u}_{i}$.

Some Joints Reach Their Limits

When certain joints reach their limits, e.g., $\left[q_{i}, q_{j}, q_{k}\right]=\left[q_{i}^{\text {limit }}, q_{j}^{\text {limit }}, q_{k}^{\text {limit }}\right]$, the corresponding diagonal elements in the matrix $\left[\mathbf{q}_{\lambda}^{*}\right]$ will be equal to zero. For example, if $q_{i}=q_{i}^{\min }$ (or $q_{i}^{\max }$ ), the diagonal element of $\left[\partial \mathbf{q}^{*} / \partial \lambda\right]_{i i}$ will be zero (i.e., $b_{i} \cos \lambda_{i}$ is zero for either $i=1, \ldots, 2 n$ then $q_{i}$ has reached a limit). Therefore, the corresponding $\frac{\partial}{\partial \mathbf{q}^{*}}\left[\Omega\left(q_{i}, \mathbf{q}^{*}\right)\right]$ is subjected to the rankdeficiency criterion.

$$
\left[\mathbf{H}_{\mathbf{q}}\right] \sim\left[\begin{array}{ccccccc}
\boldsymbol{\xi}_{q_{1}} & \ldots & \boldsymbol{\xi}_{q_{i}} & \boldsymbol{\xi}_{q_{j}} & \boldsymbol{\xi}_{q_{k}} & \ldots & \boldsymbol{\xi}_{q_{n}} \\
0 & \ldots & 1 & 0 & 0 & \ldots & 0 \\
0 & \ldots & 0 & 1 & 0 & \ldots & 0 \\
0 & \ldots & 0 & 0 & 1 & \ldots & 0
\end{array}\right]
$$

Solving the row rank deficiency condition for Eq. 7 is equivalent to solving the rank deficiency for

$$
\begin{aligned}
& {\left[\boldsymbol{\xi}_{\mathbf{q}^{*}} \not \subset\left[\boldsymbol{\xi}_{q_{i}}, \boldsymbol{\xi}_{q_{j}}, \boldsymbol{\xi}_{q_{k}}\right]\right],} \\
& q_{i}=q_{i}^{\text {limit }}, q_{j}=q_{j}^{\text {limit }}, q_{k}=q_{k}^{\text {limit }}
\end{aligned}
$$

with 
where the notation of $\not \subset$ represents the exclusion of the right matrix from the left matrix and

it represents the sub-matrix of $\left[\xi_{q^{*}}\right]$ when joint constraints

are at their limits. From the foregoing observation, the second type of singular sets are formulated. Define a new vector $\partial \mathbf{q}^{\text {limit }}=\left[q_{i}^{\text {limit }}, q_{j}^{\text {limit }}, q_{k}^{\text {limit }}\right]^{T}$ which is a sub-vector of $\mathbf{q}^{*}$ where

$$
1 \leq \operatorname{dim}\left(\partial \mathbf{q}^{\text {limit }}\right) \leq(n-3)
$$

For the case of $\operatorname{dim}\left(\partial \mathbf{q}^{\text {limit }}\right)=(n-2)$, it is noted that the solution of Eq. 7 is readily available as will be discussed in the following paragraphs. The joint coordinates can be partitioned as

$$
\mathbf{q}^{*}=\left[\mathbf{w}^{T}, \partial \mathbf{q}^{\text {limit }^{T}}\right]^{T} \text {, and } \mathbf{w} \cap \partial \mathbf{q}^{\text {limit }}=\phi
$$

Then, if $\left[\xi_{\mathbf{w}}\left(\mathbf{w}, \partial \mathbf{q}^{\text {limit }}\right)\right]$ is row rank deficient, the subJacobian $\left[\xi_{\mathbf{q}^{*}}\right]$ is also rank deficient. Let the solution for this condition be denoted by $\hat{\mathbf{p}}$, which is a constant sub-vector of $\mathbf{w}$, and $\mathbf{w}=\left[\mathbf{u}^{T}, \hat{\mathbf{p}}^{T}\right]^{T}$. The type II singularity set is defined as

$S^{(2)} \equiv\left\{\mathbf{p}=\left[\hat{\mathbf{p}} \cap \partial \mathbf{q}^{\text {limit }}\right]: \operatorname{Rank}\left[\xi_{\mathbf{q}^{*}}\left(\mathbf{w}, \partial \mathbf{q}^{\text {limit }}\right)\right]<3\right.$,

for some $\left.\hat{\mathbf{p}} \in \mathbf{w}, \operatorname{dim}\left(\partial \mathbf{q}^{\text {limit }}\right) \leq(n-3)\right\}$

As for the case of $\operatorname{dim}\left(\partial \mathbf{q}^{\text {limit }}\right)=(n-2)$, i.e., only two joint variables are allowed to vary in their ranges, $\operatorname{dim}(\mathbf{w})=2$, and the sub-matrix $\left[\xi_{w}\right]$ will be of dimension $(3 \times 2)$, i.e., already row rank deficient. For this case, there are no solutions, but the type-III singularity is

$$
S^{(3)} \equiv\left\{\mathbf{p} \in \Re^{(n-2)}: \mathbf{p} \equiv \partial \mathbf{q}^{\text {limit }}=\left[q_{i}^{\text {limit }}, q_{j}^{\text {limit }}, \ldots\right]\right\}
$$

\section{Hyperentities}

Substituting the set $\mathbf{p}_{i}$ characterized by the sets obtained in Eqs. 11, 18, and 19, into the accessible set $\boldsymbol{\xi}\left(\mathbf{q}^{*}\right)$ yields a hyperentity parametrized in terms of the remainder variables as

$$
\xi\left(\mathbf{p}_{i}, \mathbf{u}_{i}\right)=\left\{\begin{array}{c}
\text { where } \mathbf{p}_{i} \in S^{(1)} \text { of Eq. (11) } \\
\text { where } \mathbf{p}_{i}=\left[\hat{\mathbf{p}}^{T}, \partial \mathbf{q}^{\text {limit }}{ }^{T}\right]^{T} \in S^{(2)} \text { of Eq. (18) } \\
\text { where } \mathbf{p}_{i}=\partial \mathbf{q}^{\text {limit }} \in S^{(3)} \text { of Eq. (19) }
\end{array}\right.
$$

subject to the inequality constraints of the generalized coordinates $q_{i}-a_{i}-b_{i} \sin \lambda_{i}$. The vector function characterized by Eq. 20 is a hypersurface that is parametrized either in two parameters $\mathbf{u}_{i}=\left[\begin{array}{ll}q_{k} & q_{\ell}\end{array}\right]^{T}$ or three parameters $\mathbf{s}_{i}=\left[\begin{array}{lll}q_{k} & q_{\ell} & q_{m}\end{array}\right]^{T}$ such that

$\boldsymbol{\xi}(\mathbf{q})=\left\{\begin{array}{ll}\operatorname{dim}\left(\mathbf{p}_{i}\right)=3, & \rightarrow \boldsymbol{\xi}\left(\mathbf{u}_{i}\right) \\ \operatorname{dim}\left(\mathbf{p}_{i}\right)=2, & \rightarrow \xi\left(\mathbf{s}_{i}\right)\end{array}\right\}$

Hypersurfaces that are parametrized in two variables $\left(\boldsymbol{\xi}\left(\mathbf{u}_{i}\right)\right)$ have their limits prescribed by he inequality constraints and can readily be depicted. However, hypersurfaces parametrized in three variables $\left(\boldsymbol{\xi}\left(\mathbf{s}_{i}\right)\right)$ represent coupling between the joints and require further analysis.

\section{COUPLED SINGULAR BEHAVIOR}

For a 5-R manipulator and if two joints are locked, the remaining 3-DOF's end-effector may have a volume as its workspace. However, in some cases and because of the coupled singular behavior, this workspace is only a surface parametrized in three variables indicating a coupling between (e.g., a Gimbal). It was noticed that when this case occurs, at least two joints of the reduced order manipulator are coupled, and at least one configuration has has a rank deficiency of two. Since the rank-deficiency of this surface is of order one, it yields two constant generalized coordinates, i.e., oneparameter geometric entities. Curves instead of surfaces that identify the boundary will be determined.

For this case, $\boldsymbol{\xi}_{i}\left(\mathbf{s}_{i}\right): \mathbf{s}_{i} \in \mathfrak{R}^{3} \rightarrow \mathfrak{R}^{3}$, and the Jacobian of the hypersurface is

$$
\boldsymbol{\xi}_{\epsilon}=\llbracket \boldsymbol{\xi}_{\mathrm{s}} \rrbracket\left[\mathbf{S}_{\epsilon}\right]
$$

where $\varepsilon$ is the corresponding vector of slack variables $\boldsymbol{\epsilon}=\left[\begin{array}{lll}\lambda_{k} & \lambda_{\ell} & \lambda_{m}\end{array}\right]^{T}$. For a joint at its limit $\left(q_{i}^{\text {limit }}\right)$, the second block matrix of Eq. 22 is rank deficient. An elementary matrix of row operations $\mathbf{E}_{i}$ applied to $\left[\mathbf{s}_{\varepsilon}\right]$ yields a row echelon form such that

$$
\mathbf{E}_{i}\left[\mathbf{S}_{\varepsilon}\right]=\mathbf{E}_{R E}
$$

where $\mathbf{E}_{1}=\left[\begin{array}{ccc}0 & 1 & 0 \\ 0 & 0 & 1 \\ 0 & 0 & 0\end{array}\right], \quad \mathbf{E}_{2}=\left[\begin{array}{ccc}1 & 0 & 0 \\ 0 & 0 & 1 \\ 0 & 0 & 0\end{array}\right], \quad$ and $\quad \mathbf{E}_{3}=\left[\begin{array}{ccc}1 & 0 & 0 \\ 0 & 1 & 0 \\ 0 & 0 & 0\end{array}\right]$; where the subscript denotes the joint number, and $\mathbf{E}_{R E}$ is a row echelon form. This same matrix applied to $\left[\xi_{\mathrm{s}}\right]^{T}$ yields

$$
\begin{gathered}
\mathbf{E}_{i}\left[\boldsymbol{\xi}_{\mathrm{s}}\right]^{T}=\left[\begin{array}{ll}
\boldsymbol{\Lambda} & \mathbf{0}
\end{array}\right]^{T} \\
\text { where }[\boldsymbol{\Lambda}]=\left[\begin{array}{lll}
\boldsymbol{\Lambda}_{1} & \boldsymbol{\Lambda}_{2} & \boldsymbol{\Lambda}_{3}
\end{array}\right]
\end{gathered}
$$

where $\operatorname{dim}(\Lambda)=(2 \times 3)$ and $\operatorname{dim}\left(\Lambda_{j}\right)=(2 \times 1)$ for $j=1,2,3$. Applying Eq. 12 to $\llbracket \Lambda \rrbracket$ with $m=3, n=2$ variables, and a rank deficiency of $d=1$ yields $\eta=3$ 
equations to be solved simultaneously. Solutions to the three equations are singular sets denoted by $\gamma_{i}$ such that

$\gamma_{i}=\left\{\left[\begin{array}{l}D_{1} \\ D_{2} \\ D_{3}\end{array}\right]=\mathbf{0}\right.$, for $\left.\gamma_{i} \in \mathbf{s}, \gamma_{i} \cap \beta_{i}=\phi\right\}$

where $D_{1}=\left|\Lambda_{1} \Lambda_{2}\right|, \quad D_{2}=\left|\Lambda_{2} \Lambda_{3}\right|, \quad$ and $\quad D_{3}=\left|\Lambda_{1} \Lambda_{3}\right|$ subject to the joint constraints and where $\gamma_{i}$ is a subset of $\mathbf{s}$ such that $\mathbf{s}_{i}^{T}=\left[\begin{array}{ll}\gamma_{i}^{T} & \beta_{i}\end{array}\right]$, where $\beta_{i}$ is the remaining variable such that

$\xi_{i}\left(\gamma_{i}, \beta_{i}\right)=\Gamma_{i}\left(\beta_{i}\right)$

where $\Gamma_{i}\left(\beta_{i}\right)$ is a parametric curve. $\operatorname{Dim}(\Gamma(\beta))=(3 \times 1)$ represents boundary curves to the hypersurface $\boldsymbol{\xi}(\mathbf{s})$. The workspace is characterized by the hypersurfaces $\boldsymbol{\xi}(\mathbf{u})$ and $\boldsymbol{\xi}(\mathbf{s})$ subject to $\Gamma(\beta)$. The determination of its boundary is addressed in the following section.

\section{PERTURBATION METHOD TO DETERMINE THE BOUNDARY}

Since hypersurfaces extend internal and external to the workspace envelope, it is necessary to identify regions (surface patches) of these hypersurfaces that are on the boundary, whether the external boundary or a void.

The curves resulting from the intersection of hypersurfaces divide each surface into many regions. An algorithm developed by the authors (Abdel-Malek and Yeh 1996, 1997c) is implemented to identify these regions whereby curves of intersection are traced. In fact, these curves represent singular trajectories of the end-effector at which the manipulator looses at least two degrees of freedom (coupled singularities). The intersection of two singular curves identify the so-called bifurcation point.

To determine if a region is internal, a perturbation method is employed. Consider a point $\mathbf{q}^{c}$ on a hypersurface but not on the boundary, i.e., $q_{i}^{L}<q_{i}^{c}<q_{i}^{U}$. At $\boldsymbol{\xi}\left(\mathbf{q}^{c}\right)$, the velocity of the end-effector is given by

$[\dot{\xi}]=\left[\xi_{\mathbf{q}^{*}}\right]\left[\mathbf{q}_{\lambda}^{*}\right][\dot{\lambda}]$

On a singular surface, the term $\left.\left[\boldsymbol{\xi}_{\mathbf{q}^{*}} \mathbf{q}_{\lambda}^{*}\right]\right|_{\mathbf{q}_{o}^{*}, \lambda_{o}}$ is rankdeficient. Multiplying both sides of Eq. 28 by $\mathbf{N}_{o}^{T}$ (the basis of the null space of $\boldsymbol{\xi}_{\mathbf{q}^{*}} \mathbf{q}_{\lambda}^{*}$ ) yields

$\mathbf{N}_{o}^{T} \dot{\xi}=\mathbf{N}_{o}^{T} \boldsymbol{\xi}_{\mathbf{q}^{*}}\left(\mathbf{q}^{c}\right) \mathbf{q}_{\lambda}^{*} \dot{\lambda}=0$

Since $\mathbf{N}_{o}^{T}$ is a constant vector at $\mathbf{q}^{c}$, the left hand side of Eq. 29 characterizes the equation of a plane in $\mathfrak{R}^{3}$ as
$N_{1} \dot{x}+N_{2} \dot{y}+N_{3} \dot{z}=0 \quad$ where $\mathbf{N}_{o}=\left[\begin{array}{lll}N_{1} & N_{2} & N_{3}\end{array}\right]^{T}$ is indeed a vector normal to the tangent plane of the singular surface at $\mathbf{q}^{c}$. Indeed, for any value of $\dot{\mathbf{q}}$, all resulting velocity vectors $\dot{\boldsymbol{\xi}}$ will lie on a plane which has $\mathbf{N}_{o}^{T}$ as its normal. Two observations can be made:

(i) The basis of the null space of $\left[\xi_{\mathbf{q}^{*}} \mathbf{q}_{\lambda}^{*}\right]^{T}$ is the vector normal to the singular surface at $\mathbf{q}^{c}$.

(ii) For any given joint velocity vector $\dot{\mathbf{q}}$, the velocity of the end-effector is either tangent to the singular surface or zero, i.e., the normal component of the end-effector velocity is always zero, i.e., $v_{n}=\mathbf{N}_{o} \cdot \dot{\boldsymbol{\xi}}=\mathbf{N}_{o}^{T} \dot{\boldsymbol{\xi}}=0$ (this result is reported by Haug et al. 1996 using a different approach).

For this normal, two points along the normal on each side of the surface can be found as

$$
\boldsymbol{\xi}^{p_{1,2}}=\boldsymbol{\xi}\left(\mathbf{q}^{c}\right) \pm \mathbf{N} \partial \vartheta
$$

where $\partial \vartheta$ is a small variation from $\boldsymbol{\xi}\left(\mathbf{q}^{c}\right)$ (e.g., $\partial \vartheta=0.1$ ). If both points $\xi^{p_{1}}$ and $\xi^{p_{2}}$ satisfy the constraint equation, then the region for which $\boldsymbol{\xi}\left(\mathbf{q}^{c}\right)$ belongs is internal to the boundary. Performing this test on all regions yields boundary surface patches defined by the equations of $\boldsymbol{\xi}\left(\mathbf{q}^{c}\right)$ and bound by the numerical curves.

\section{BIFURCATION POINTS, VOID DETERMINATION, AND COMPUTING THE VOLUME}

A cross section of the workspace at any elevation can be computed by numerically intersecting each hypersurface with a plane. The same algorithm used above to identify regions is employed to trace intersection curves.

Bifurcation points identified by Haug et al. (1996) can now be explained as the intersection of at least two hypersurfaces. Indeed, continuation lines traced in that work are in effect the curves of intersection between two hypersurfaces. If three hypersurfaces intersect at a point, the rank deficiency of the Jacobian at this point is of order two. The computation of tangents at these points becomes more difficult as the number of surfaces augments. Void determination using the presented method becomes a simple task as the perturbation method performed on an internal region of a hypersurface reveals whether this region is a boundary. If it is a boundary while inside the external boundary, then this region envelops a void. An example presented below illustrates the determination of voids. Because hypersurfaces are in closed form, their intersection with a cutting plane can be computed at any elevation. A number of cutting planes are introduced, and their intersection with the hypersurfaces computed. The volume is computed by 
determining the area enclosed by the boundary and discretely integrating over the cross sectional areas.

\section{EXAMPLES EXAMPLE 1: A 5-DOF RRPRR MANIPULATOR}

Consider the 5-DOF manipulator shown in Fig. 1.

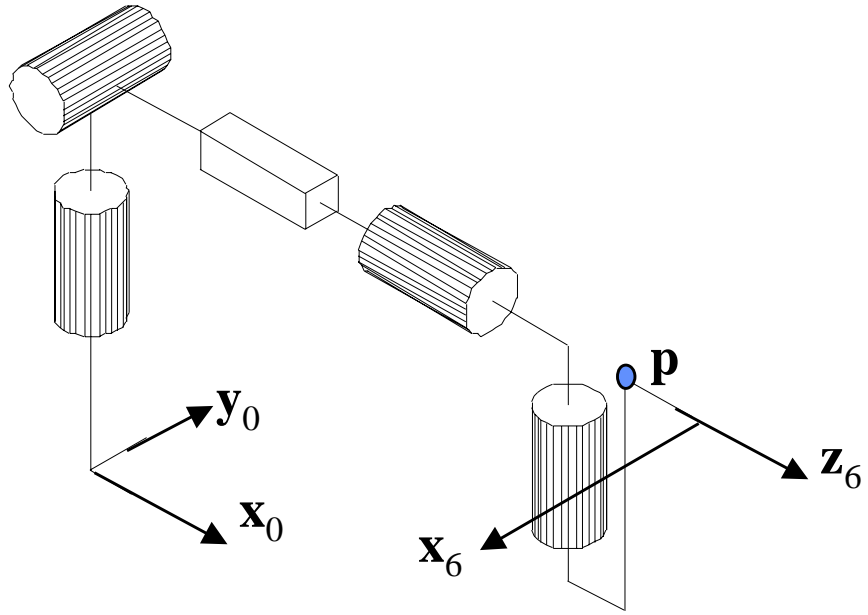

Fig. 1 A 5-DOF RRPRR manipulator

Joints are constrained as $0 \leq q_{1} \leq 2 \pi$, $-\pi / 4 \leq q_{2} \leq 5 \pi / 4, \quad 0 \leq q_{3} \leq 5, \quad 0 \leq q_{4} \leq 2 \pi, \quad$ and $-\pi / 2 \leq q_{5} \leq \pi / 2$. Using Denavit-Hartenberg, the position vector of point $\mathbf{p}$ in Fig. 1 is determined as

$\boldsymbol{\xi}=\left[\begin{array}{c}-5 \sin q_{5} \cos q_{1} \sin q_{2} \sin q_{4}-5 \sin q_{5} \sin q_{1} \cos q_{4}+ \\ -5 \sin q_{5} \sin q_{1} \sin q_{2} \sin q_{4}+5 \sin q_{5} \cos q_{1} \cos q_{4}+ \\ 5 \cos q_{2} \sin q_{4} \sin q_{5}+5 \sin q_{2} \cos q_{5}+\end{array}\right.$

$$
\left.\begin{array}{c}
5 \cos q_{1} \cos q_{2} \cos q_{5}+15 \cos q_{1} \cos q_{2}+q_{3} \cos q_{1} \cos q_{2} \\
5 \sin q_{1} \cos q_{2} \cos q_{5}+15 \sin q_{1} \cos q_{2}+q_{3} \sin q_{1} \cos q_{2} \\
15 \sin q_{2}+q_{3} \sin q_{2}+25
\end{array}\right]
$$

The criteria introduced in Sec. (2) is applied to Eq. (31) yielding the following singular sets.
$\mathbf{p}_{1}:\left\{q_{2}=\pi / 2, q_{4}=0\right.$, and $\left.q_{5}=\pi / 2\right\}$
$\mathbf{p}_{2}:\left\{q_{2}=-\pi / 4, q_{4}=0\right.$, and $\left.q_{5}=0\right\}$,
$\mathbf{p}_{3}:\left\{q_{2}=-\pi / 4, q_{4}=\pi / 2\right.$, and $\left.q_{5}=\pi / 2\right\}$
$\mathbf{p}_{4}:\left\{q_{2}=-\pi / 4, q_{4}=3 \pi / 2\right.$, and $\left.q_{5}=\pi / 2\right\}$
$\mathbf{p}_{5}:\left\{q_{3}=0, q_{4}=0\right.$, and $\left.q_{5}=0\right\}$
$\mathbf{p}_{6}:\left\{q_{2}=\pi / 2, q_{3}=0\right.$, and $\left.q_{4}=0\right\}$,
$\mathbf{p}_{7}:\left\{q_{3}=5, q_{4}=0\right.$, and $\left.q_{5}=0\right\}$
$\mathbf{p}_{8}:\left\{q_{2}=\pi / 2, q_{3}=5\right.$, and $\left.q_{4}=0\right\}$,

$\mathbf{p}_{9}:\left\{q_{2}=-\pi / 4, q_{3}=0\right.$, and $\left.q_{4}=\pi / 2\right\}$

$\mathbf{p}_{10}:\left\{q_{2}=-\pi / 4, q_{3}=5\right.$, and $\left.q_{4}=\pi / 2\right\}$

$\mathbf{p}_{11}:\left\{q_{3}=0\right.$ and $\left.q_{5}=\pi / 2\right\}, \mathbf{p}_{12}:\left\{q_{3}=5\right.$ and $\left.q_{5}=\pi / 2\right\}$

Note that hypersurfaces due to $\mathbf{p}_{11}$ and $\mathbf{p}_{12}$ are parametrized in three variables $\left(q_{1}, q_{2}\right.$, and $\left.q_{4}\right)$ indicating coupled singular behavior. A cross section of the workspace of the 5-DOF manipulator depicting all singular surfaces is shown in Fig. 2.

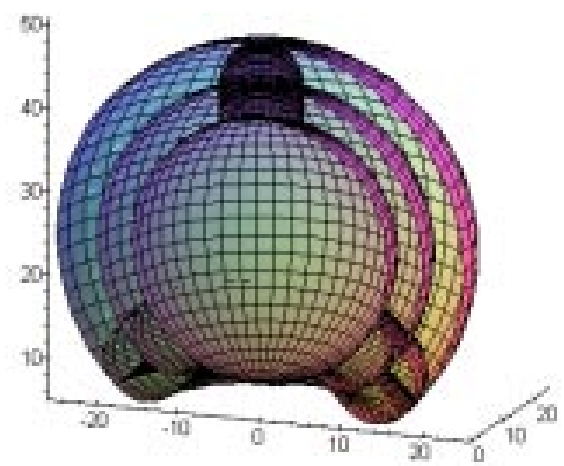

Fig. 2 A cross-section of the workspace of the RRPRR Manipulator

\section{Example 2: 4-DOF Manipulator-Calculating the Workspace Volume}

Consider the 4-DOF manipulator shown in Fig. 3.

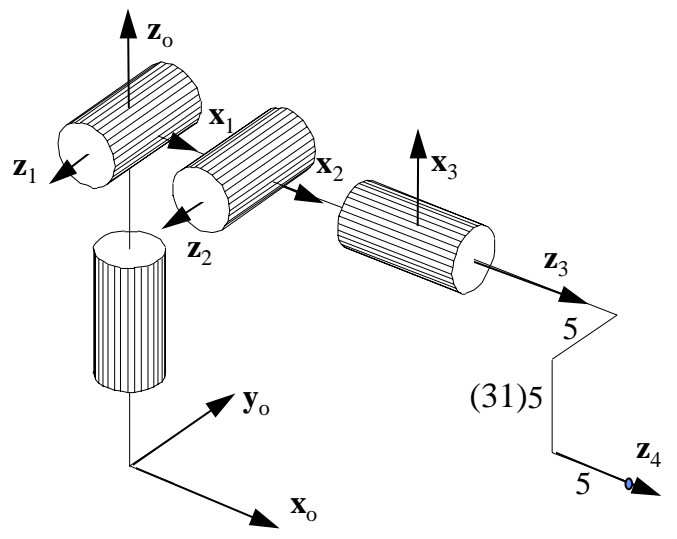

Fig. 3

There are $\mathbf{p}_{i} ; i=1 . . .36$ singular sets. Cross sections at different z-values are shown in Fig. 4. 


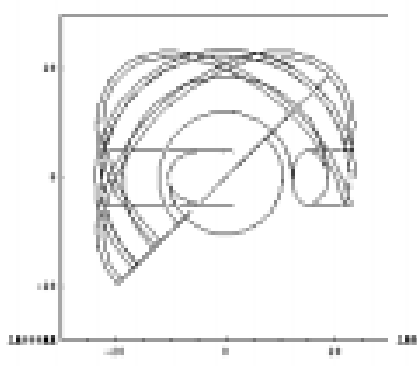

Fig. 4. (a) at $z_{i}=-2$

$$
\text { (f) at } z_{i}=28
$$

The area for each cross-section is computed and entered in Table 1.

\section{TABLE 1}

The computed area for each cross section

\begin{tabular}{|l|l|l|l|l|l|l|l|}
\hline$\partial z_{i}$ & 0 & 1 & 3 & 5 & 8 & 10 & 12 \\
\hline $\mathrm{A}$ & 673 & 786 & 967 & 1129 & 1293 & 1442 & 1553.8 \\
\hline
\end{tabular}

\begin{tabular}{|l|l|l|l|l|l|l|l|}
\hline$\partial z_{i}$ & 14 & 16 & 18 & 20 & 22 & 24 & 26 \\
\hline $\mathrm{A}$ & 1774 & 1775 & 2028 & 2392 & 2365 & 2323 & 2266 \\
\hline
\end{tabular}

\begin{tabular}{|l|l|l|l|l|l|l|l|}
\hline$\partial z_{i}$ & 28 & 30 & 32 & 34 & 36 & 38 & 40 \\
\hline $\mathrm{A}$ & 2153 & 2041 & 1871 & 1690 & 1481 & 1238 & 954 \\
\hline
\end{tabular}

\begin{tabular}{|l|l|l|l|l|l|l|l|}
\hline$\partial z_{i}$ & 42 & 44 & 45 & 45.5 & -2 & -2.5 & -2.8 \\
\hline $\mathrm{A}$ & 2153 & 2041 & 1871 & 1691 & 338 & 199 & 53 \\
\hline
\end{tabular}

The cross-sections are connected using a mesh based on a trapezoidal algorithm and the volume is computed to $V_{\text {trapez }}=71841.7 u^{3}$, where $u$ is a unit, while based on spline algorithm, the volume is computed to $V_{\text {spline }}=72149 u^{3}$.

\section{EXAMPLE 3: A 5-DOF RPPRR with Voids}

Consider the 5-DOF manipulator shown in Fig. 5, having joint limits as $\quad-153^{\circ} \leq q_{5} \leq 128^{\circ}, \quad 0^{\circ} \leq q_{4} \leq 330^{\circ}$, $0 \leq q_{3} \leq 4.5,0 \leq q_{2} \leq 2.8$, and $0 \leq q_{1} \leq 240^{\circ}$.

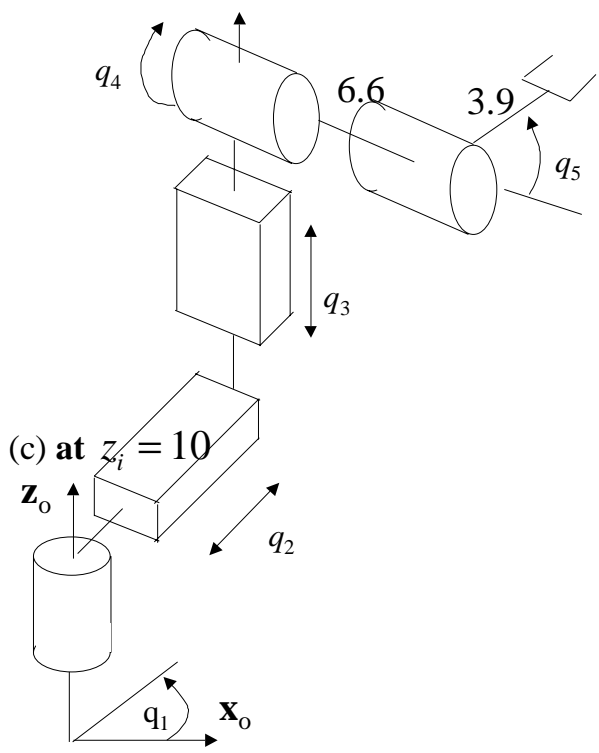

Fig. 5. A 5-DOF RPPRR manipulator

The perturbation method applied to each surface region identifies the boundary as shown in Fig. 6, where an internal boundary to a void is also identified.

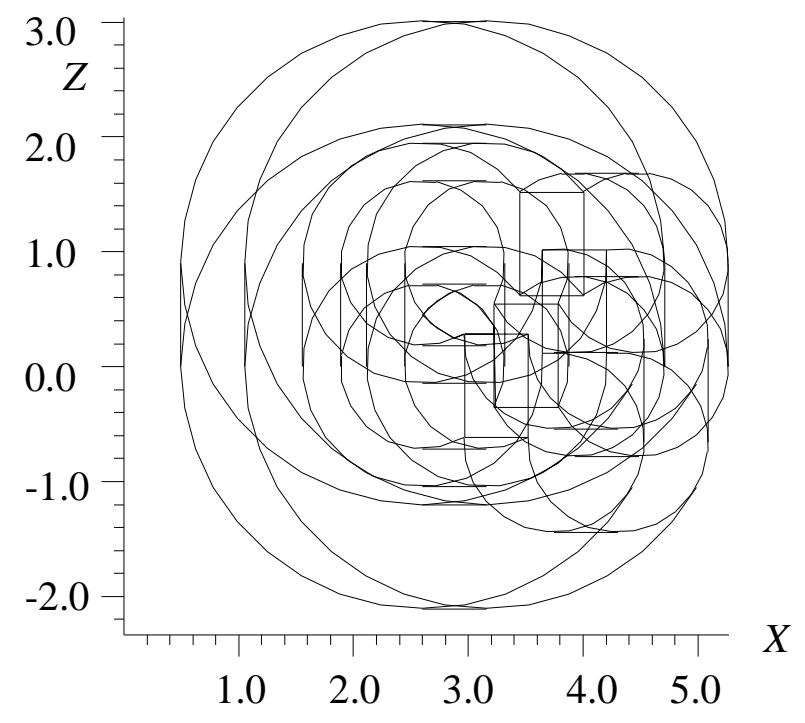




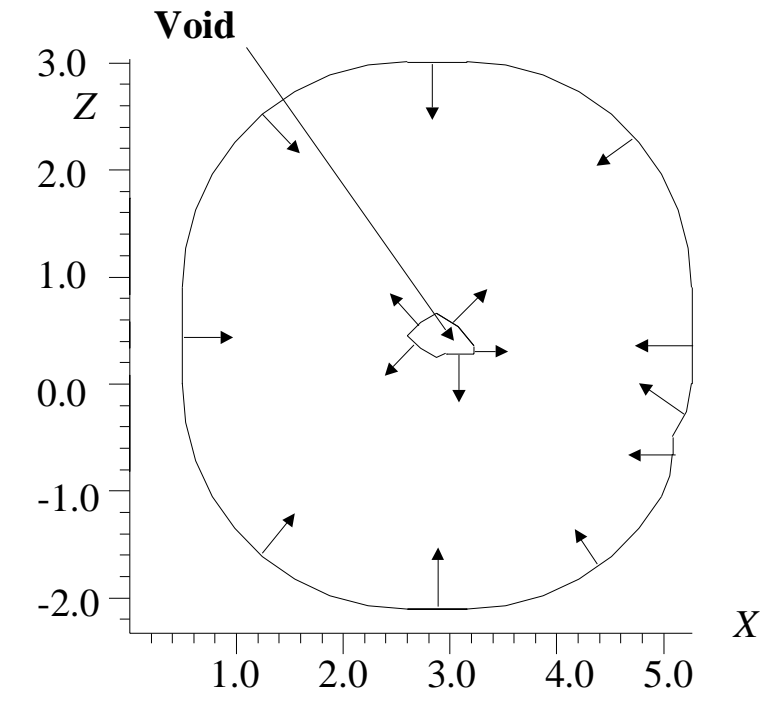

Fig. 6. (a) Cross-section of the RPPRR manipulator at $q_{1}=\pi / 2$ (b) Void determination

\section{Example 4: The 5R General Manipulator}

Consider the 5R serial arm shown in Fig. 8 where $\alpha_{i}=\pi / 3$, $d_{i}=1$ where $i=1, \ldots, 5, a_{1}=3, a_{2}=4, a_{3}=3, a_{4}=4$ and $a_{5}=3$. The cross section at $z=0.0$ is shown in Fig. 9 .

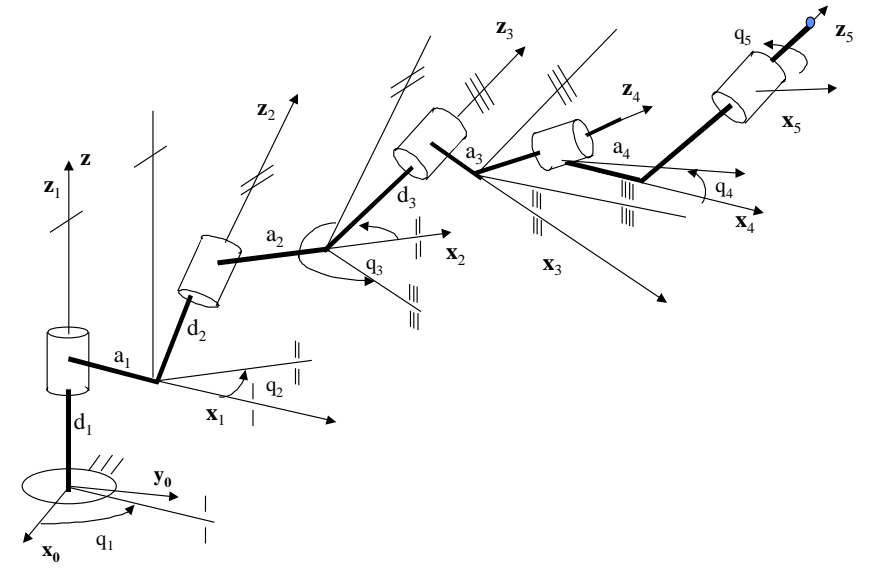

Fig. 8 The general 5-revolute Manipulator

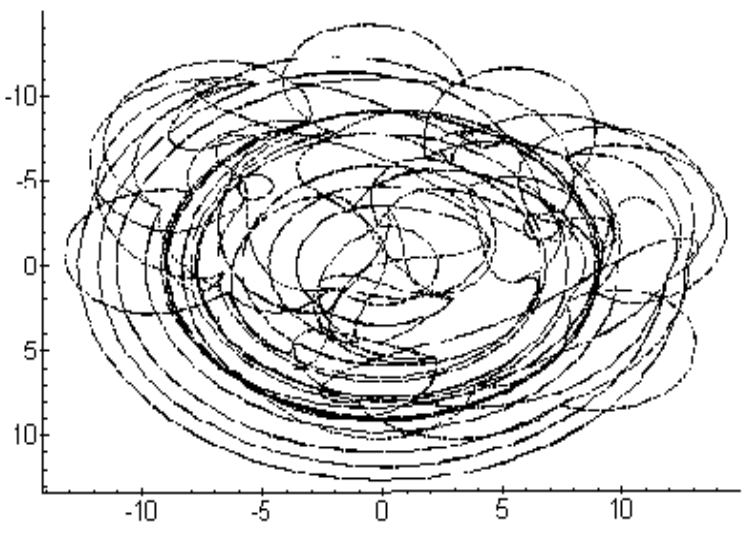

Fig. 9 A cross section of the general 5R manipulator

\section{CONCLUSIONS}

A general formulation for determining boundary surface patches in closed form to 5DOF manipulators has been presented. The workspace constraint function was formulated in terms of generalized coordinates including inequality constraints imposed on each joint. It was shown that Jacobian rank-deficiency conditions usually applied in robotics analysis to determine degenerate conditions, are employed here to generate constant singular sets and to identify coupled singular behavior. It was also shown that hypersurfaces in parametric form based on these singular sets exist internal, external, or may extend from the internal to the external of the workspace envelope. It is emphasized that these hypersurfaces are characterized by parametric equations where the parameters are joint variables. It was observed that those surfaces that are parametrized in three variables exhibit singular behavior that can be used to determine their boundaries in terms of parametric space curves.

Coupled singular behavior for hypersurfaces parametrized in three variables was addressed and has been shown to provide solutions to a class of problems identical to the case of the Gimbals mechanism.

Results pertaining to the determination of the workspace, envelope volume, bifurcation analysis, and cross sectional views of the workspace were presented. Validation examples, were illustrated to demonstrate the applicability of the formulation to a wide range of problems.

One may conclude that the solution to these examples are possible only because hyperentities are identified in closed form. To the authors' belief, these are the only reported results that have yielded closed form equations to surface patches of the workspace envelope.

\section{ACKNOWLEDGMENTS}

This research was funded by the US Army Tank Automotive Research Center (TACOM) through the ARC (Department of 
Defense contract number DAAE07-94-C-R094) at the Iowa Center for Computer-Aided Design.

\section{REFERENCES}

Abdel-Malek, K. and Yeh, H.J., 1996, "Determining Intersection Curves Between Surfaces of Two Solids," Computer Aided Design, Vol. 28 (6/7) pp. 539-549.

Abdel-Malek, K. and Yeh, H.J., 1997a "Geometric Representation of the Swept Volume Using Jacobian Rank-Deficiency Conditions," Computer Aided Design, v29(6), pp. 457-468.

Abdel-Malek, K. and Yeh, H.J., 1997b, "Analytical Boundary of the Workspace for General 3-DOF mechanisms," International Journal of Robotics Research. v16(2), pp. 112.

Abdel-Malek, K and Yeh, H J, 1997c, "On the Determination of Starting Points for Parametric Surface Intersections," Computer-Aided Design, Vol. 29(1) pp. 21-35.

Abdel-Malek, K., Adkins, F., Yeh, H.J., and, Haug, E.J, 1997, "On the Determination of Boundaries to Manipulator Workspaces," Robotics and Computer-Integrated Manufacturing, Vol. 13, No. 1, pp.1-15.

Ceccarelli, M. and Vinciguerra, A., 1995, "On the Workspace of General 4R Manipulators," International Journal of Robotics Research, Vol. 14, No. 2, pp. 152-160.

Ceccarelli, M., 1995, "A Synthesis Algorithm for ThreeRevolute Manipulators by Using an Algebraic Formulation of Workspace Boundary," ASME J. of Mechanical Design, Vol. 117, No. 2(A), pp. 298-302.

Denavit, J., and Hartenberg, R.S., 1955, "A Kinematic Notation for Lower-pair Mechanisms Based on Matrices," Journal of Applied Mechanics, ASME, Vol. 22, pp. 215221.

Emiris, D.M., 1993, "Workspace Analysis of Realistic Elbow and Dual-elbow Robot," Mechanisms and Machine Theory, Vol. 28, No. 3, pp. 375-396.

Fu, S., Gonzalez, J, and Lee, S., 1987, Robotics: Control, Sensing, Vision, And Intelligence. McGraw-Hill, Inc., New York.

Haug, E.J., Wang, J.Y., and Wu, J.K., 1992, "Dextrous Workspaces of Manipulators: I. Analytical Criteria," Mech. Struct. and Mach., Vol. 20, No. 3, pp. 321-361.

Haug, E.J., Luh, C.M., Adkins, F.A., and Wang, J.Y., 1996, "Numerical algorithms for mapping boundaries of manipulator workspaces," ASME Journal of Mechanical Design, 118, pp. 228-234.

Kumar, V., 1985, Robot Manipulators-Workspaces and Geometric Dexterity, Masters Thesis, The Ohio State University.

Luh, C. M. Adkins, F. A. Haug, E. J. and Qiu, C.C., 1996, "Working Capability of Stewart Platforms," ASME Journal of Mechanical Design, Vol. 118, pp. 220-227.

Pennock, G.R. and Kassner, D.J., 1993, "The Workspace of a General Planar Three-Degree-of-Freedom Platform-Type
Manipulator," ASME Journal of Mechanical Design, Vol. 115 , pp. 269-276.

Spanos, J. and Kohli, D., 1985, "Workspace Analysis of Regional Structures of Manipulators," Journal of Mechanisms, Transmissions, and Automation in Design, 107:216-222.

Wang, J.Y. and Wu, J.K., 1993, "Dexterous Workspaces of Manipulators, Part 2: Computational Methods," Mechanics of Structures and Machines, v21(4), pp. 471506.

Zhang, S.J., Sanger, D.J., and Howard, D., 1996, “Workspaces of a Walking Machine and their Graphical Representation. Part I: kinematic workspaces," Robotica, 14, pp. 71-79 